\title{
GROSSESSES OBTENUES PAR FÉCONDATION IN VITRO AVEC SPERME ÉPIDIDYMAIRE.
}

\author{
M.Cognat*, J.-Cl. Czyba**, J.-F. Guérin**, Cl. Mathieu**, M.-Cl. Pinatel** \\ * Centre Fertily - Lyon - 10, Av. des Frères-Lumière - 69008 LYON. \\ ** Centre de Biologie de la Reproduction - 6, place d'Arsonval - Hôpital Edouard Herriot - 69008 LYON.
}

PREGNANCIES OBTAINED AFTER IN VITRO FERTILIZATION (IVF) USING EPIDIDYMAL SEMEN. Excretory azoospermia, or rather some of its anatomoclinical forms, represents a new indication of IVF using epididymal semen are reported (our overall statistics concerns 15 attempts). Practical applications of the method are discussed. Key-words : epididymis, epididymal semen, pregnancy, IVF, excretory azoospermia. Andrologie, 1991, 1 : 20-21

Depuis plusieurs années, nous développons les méthodes de prélèvement de sperme épididymaire en vue de Fécondation In Vitro (FIV). Nous rapportons ici notre expérience.

\section{MATERIEL ET METHODES}

Sujets : 13 couples répondant aux indications suivantes (11 obstructions d'origine congénitale par aplasie plus ou moins étendue et 2 oblitérations post-inflammatoires) ont été soumis à 15 essais.

Technique de prélèvement : les spermatozoïdes épididymaires sont prélevés sur la partie la plus distale et accessible du tube, par une incision transversale suivie du recueil du liquide épididymaire plus ou moins abondant, plus ou moins sous tension, par micro-aspiration, au besoin sous amplification optique, utilisant des seringues à Insuline branchées sur un cathéter veineux type Abbott-cath $\mathrm{N}^{\circ} 4$ ou un cathéter lympho-graphique - l'épididyme étant soumis à une pression douce et continue et parfois à de véritables massages. Cette seule incision distale est parfois suffisante pour recueillir une quantité adéquate de spermatozoïdes fécondants, et dans la mesure du possible le prélèvement est fait unilatéralement pour préserver les possibilités de recueils ultérieurs.

Bien souvent en fait, les incisions doivent être multipliées, successives, en remontant vers la tête épididymaire et les canaux efférents - l'expérience prouve que c'est le plus souvent à ce niveau, très haut sur la tête de l'épididyme, que la collecte des spermatozoïdes est la plus fructueuse, au moins en qualité. $(1,3,6,7)$
Préparation du sperme : la préparation des spermatozoïdes est faite immédiatement par le biologiste auquel sont confiées en salle d'opération les micro-pipettes - il utilise soit la migration sédimentation, soit mieux le gradient discontinu de PERCOLL à 6 couches $(40 \%$ $100 \%$ ) ou mieux encore le gradient discontinu simplifié de PERCOLL à 6 couches $(40 \%$ et $70 \%) .(4,1)$.

\section{RESULTATS}

Le prélèvement épididymaire a été une fois négatif : aucun spermatozoïde n'a pu être recueilli en raison de l'aspect anatomique : têtes épididymaires flottantes non connectées avec le pôle supérieur du testicule, et 14 fois positif avec mobilité nulle 2 fois, mauvaise 5 fois, et bonne avec trajets directs 7 fois.

La ponction ovocytaire a été positive 14 fois, ayant permis le recueil de 4 à 26 ovocytes par ponction, soit 158 ovocytes au total.

La fécondation a été obtenue lors de 6 essais, donnant 25 embryons au total soit un taux de fécondation par ovocyte de $15,8 \%$. Ainsi 6 transferts ont pu avoir lieu aboutissant à un taux de grossesses par tentative de $14 \%$ et un taux de grossesses par transfert de $33 \%$ (si tant est que l'on puisse parler pourcentages à partir de ces chiffres relativement peu importants).

Voici plus en détail les deux observations des grossesses obtenues par fécondation in vitro avec sperme épididymaire :

- $1^{\text {er }}$ dossier : il concerne une azoospermie excrétoire par anomalie localisée des anses épididymo-déférentielles. Le mari était âgé de 26 ans, la femme de 24 ans. Une anastomose épididymo-déférentielle s'était soldée par un échec.La première tentative de fécondation in vitro avec sperme épididymaire n'a donné qu'un seul embryon à développement tardif, et pas de grossesse évolutive. Sur une deuxième tentative de fécondation in vitro avec sperme épididymaire, une grossesse est mise en route en juillet 89 après stimulation par HMG suite à un blocage hypophysaire par DTRP6-LHRH (DECAPEPTYL 3.75 retard) en protocole long, ayant permis d'obtenir 15 ovocytes et 10 embryons dont 6 ont été congelés et 4 transférés immédiatement permettant une grossesse gémellaire avec accouchement par césarienne (un garçon et une fille normaux) en Avril 90.

$-2^{\mathrm{c}}$ dossier : il concerne une azoospermie excrétoire par aplasie bilatérale étendue de la queue de l'épididyme et du déférent. Le mari était âgé de 43 ans, la femme de 36 . La grossesse évolutive a été obtenue sur une première tentative de fécondation in vitro avec sperme épididymaire en février 90 sur un programme comportant une stimulation par les HMG après blocage hypophysaire par le DTRP6-LHRH (DECAPEPTYL 3.75 retard) en protocole long ayant permis d'obtenir 5 ovocytes qui ont développé 3 embryons. Au début de la grossesse, 3 sacs se sont développés, mais la grossesse s'est poursuivie en gemellaire de bonne vitalité aboutissant à un accouchement par césarienne (deux garçons normaux) en Novembre 90.

\section{DISCUSSION}

Le résultat de notre expérience, qui a toujours comporté un transfert classique par voie transcervicale (comme l'avait préconisé TEMPLE-

SMITH (9) tendrait à prouver l'inutilité du recours au transfert plus invasif par coelioscopie de type ZIFT ou TET préconisé par RICARDO

ASCH et SHERMAN SILBER (1) (8).

Ces succès obtenus avec des spermatozoïdes prélévés en général sur la tête, prouvant que certains de ces spermatozoïdes sont fécondants, contre-disent-ils les conceptions biologiques classiques depuis longtemps établies, à savoir que le transit épididymaire et déférentiel complet est nécessaire pour que le spermatozaïde acquiert mobilité, maturité et surtout fécondance (5) ?. Il semble que non, car on peut affirmer maintenant que les choses ne se passent pas de la même manière dans les conditions physiologiques, lorsqu'il y a écoulement normal et permanent de fluide épididymaire, et dans les situations d'obstruction, quelles qu'en soit d'ailleurs la cause : lorsqu'il y a écoulement normal, les spermatozoïdes sont normalement immobiles dans la tête, et ne deviennent effectivement mobiles et fécondants que dans le déférent, alors que s'il y a obstruction, ces mêmes spermatozoïdes épididymaires sont déjà souvent mobiles dans la tête - ces constatations n'expliquent 
cependant pas le phénomène.

Les indications de la méthode sont :

- essentiellement l'obstruction congénitale du déférent et/ou de l'épididyme par aplasie plus ou moins étendue,

- et l'oblitération post-inflammatoire du tractus épididymo-déférentiel (après MST), non opérable ou après échec d'une anatomose épididymodéférentielle.

(Ces deux indications impliquant bien entendu que la spermatogénèse ait été véréfiée normale et que la tête de l'épididyme ou une partie de celleci au moins soit préservée et accessible.)

\section{CONCLUSION}

1. - Le type de traitement in vitro des spermatozoïdes se révèle d'une grande importance pour la fécondation,

2. - Les premiers résultats positifs ( 2 grossesses gémellaires avec enfants normaux) que nous présentons confirment la possibilité d'éviter le recours au sperme de donneur pour certains de ces couples dont l'azoospermie était jusqu'à considérée comme définitivement incurable, après l'abandon des spermatocèles dont les résultats ont été décevants.

\section{REFERENCES}

1 - Asch R, Ord T, Balmaceda J, Patrizio P, Marel E, Silber $S$. Infertility due to congenital absence of the vas deferens. Results of a new treatment based on epididymal sperm retrieval, in vitro fertilization, and tubal embryo transfer. International Congress of IVF and assisted procreations - Jerusalem - Mai 1989.

2 - Cognat M, Zaroukian A, Bardet J, Czyba J.C, Guérin J.F, Pinatel M.Cl, Lornage J, Regnier-Vigouroux G. Grossesses-Fiv avec sperme épididymaire. A propos de deux succès. Congrès de la S.A.L.F., Sousse 6-8 septembre 1990

3 - Cognat M, Delafontaine D. Utilisation du sperme épididymaire et déférentiel dans un programme de procréation médicalement assistée. 18èmes journées de Fertilité-Orthogénie. Les Nouveautés en Fertilité - Paris 10, 11, 12 novembre 1990.

4 - Guérin J.F, Mathieu C.L, Lornage J, Pinatel M.Cl, and Boulieu D. Improvement of survival and fertilizing capacity of human spermatozoa in a IVF programm by selection on discontinuous Percoll gradients. Human Reproduction. 1989, $4: 798-804$

5 - Orgebin-Crist M.C. Physiologie de l'épididyme et maturation du sperme : état actuels des connaissances. Contraception-Fertilité-Sexualité - 1986, 14 : 487-495.

6- Schoysman R, Bertin G, Van Der Zwalmen P et Segal L. Utilisation du sperme épididymaire dans un programme de FIV; In - Stérilité Masculine et Procréations Médicalement Assistées, Progrès en
Andrologie $n^{\circ} 3$, Y. Englert, J.F Guerin, P. Jouannet eds, Doin, Paris, 1989 : 137-148.

7 - Silber S. Apparent Fertility of Human Spermatozoa from the Caput Epididymis. Andrologia, $1989,10: 263-269$.

8 - Silber S, Balmaceda J, Borrero C, Ord T, Asch R. Pregnancy with sperm aspiration from the proximal head of the epididymis : a new treatment for congenital absence of the vas deferens. Fertility Sterility, 1988, 50 : 525-528.

9 - Temple-Smith P.D, Southwick G.J., Yates C.A, Trounson A.O, De Kretser D.M. Human pregnancy by in vitro fertilization (IVF) using sperm aspirated from the epididymis. Journal of in vitro fertilization and embryo transfert, 1985, $2: 119-122$.

RESUME : L'azoospermie excrétoire, ou plutôt certaines de ses formes anatomocliniques, représente une nouvelle indication de la fécondation in vitro. Deux grossesses obtenues par fécondation in vitro avec spermatozoïdes épididymaires sont l'occasion de faire une revue des applications pratiques de la méthode, et de présenter notre statistique globale reposant sur 15 essais. Mots clés : épididyme, sperme épididymaire, grossesse, FIV, azoospermie excrétoire.

Andrologie, 1991, 1 : 20-21

\section{NOUVEAUTÉS THÉRAPEUTIQUES EN ANDROLOGIE}

Une séance organisée par W.B. SCHILL et M. SCHOUMAN le Samedi 30 Novembre à $14 \mathrm{~h}$ va leur être consacrée au cours du congrès de Strasbourg de la Société d'Andrologie de Langue Française.

L'exposé introductif de G. HAIDL (Giessen) précisera l'état actuel des possibilités thérapeutiques en Andrologie.

Puis M. ETCHEVERRY (Bordeaux) fera le point de l'apport de la thermothérapie dans le traitement de l'adénome prostatique. Cette technique récente voudrait éviter ou reculer l'échéance chirurgicale chez certains patients. Tandis que plusieurs fabricants s'emploient à promouvoir activement leur nouveau matériel, l'Association Française d'Urologie a mis en place un programme multicentrique pour l'évaluer objectivement. M. ETCHEVERRY y participe

J. BUVAT terminera la première partie de cette session en faisant le point des injections intra- caverneuses de Prostaglandine El. Cela fait moins de 10 ans qu'ont été découverts les effets spectaculaires de la Papavérine sur l'érection. Seule ou associée à la Phentolamine, cette drogue représente à l'évidence un apport diagnostique et thérapeutique inestimable. La possibilité des complications sérieuses s'est cependant révélée avec le temps. Des alphabloqueurs développés ultérieurement, comme le Moxisylyte, n'ont pas ces inconvénients, mais ne sont pas suffisamment puissants pour tous les cas. La Prostaglandine E1, composé qui pourrait participer à l'érection naturelle, allie puissance et innocuité, mais nous verrons qu'au moins pour l'instant, l'utilisation de cette drogue n'est pas sans inconvénient

Un entracte de $45 \mathrm{~mm}$ sera consacré à une présentation orale des meilleurs posters du congrès.

La $2^{\text {emc }}$ partie de la session sera consacrée à l'infertilité masculine. J. BELAISCH (Paris) a accepté la gageure de faire le point de l'intérêt des traitements médicamenteux dans l'oligospermie idiopathique.

L'exposé de K. DIEDRICH (Bonn) précisera les indications andrologiques des reproductions assistées, et sera une excellente introduction aux 3 communications suivantes:

- micro-injections de spermatozoïdes, traité par J.Ph. WOLF (Paris), qui en a acquis une solide expérience au sein de l'équipe de P. JOUANNET, - prélèvement micro-chirurgical des spermatozoïdes en vue d'insémination, présenté par A.D. WEISKE (STUTTGART),

- enfin Fécondation In Vitro après prélèvement épididymaire ou déférentiel des spermatozoïdes, traité par J.F. GUERIN (Lyon).

Ces 5 communications seront suivies de $30 \mathrm{mn}$ de discussion.

M. SCHOUMAN 